\title{
Valores eletrocardiográficos em saguis-de-tufo-preto (Callithrix penicillata) ${ }^{1}$
}

\author{
Amália T. Giannico $^{2 *}$, André T. Somma ${ }^{2}$, Rogério R. Lange ${ }^{2}$, James N.B.M. Andrade ${ }^{3}$, \\ Leandro Lima ${ }^{2}$, Andressa C. Souza ${ }^{2}$ e Fabiano Montiani-Ferreira ${ }^{2}$
}

\begin{abstract}
Giannico A.T., Somma A.T., Lange R.R., Andrade J.N.B.M., Lima L., Souza A.C. \& Montiani-Ferreira F. 2013. [Electrocardiographic values in marmosets (Callithrix penicillata).] Valores eletrocardiográficos em saguis-de-tufo-preto (Callithrix penicillata). Pesquisa Veterinária Brasileira 33(7):937-941. Departamento de Medicina Veterinária, Universidade Federal do Paraná, Rua dos Funcionários 1540, Curitiba, PR 80035-050, Brazil. E-mail: amaliagiannico@uol.com.br

Knowledge of cardiac parameters in experimental animal models is essential for comparative medical research. Our study aimed to establish electrocardiographic parameters and reference values for marmosets (Callithrix penicillata). Nineteen healthy adult marmosets were used. A combination of tiletamine and zolazepam was used for chemical restrained before performing electrocardiography (ECG). A computerized ECG machine was used. Values of the ECG parameters were a mean heart rate of $264 \pm 74$ beats $/ \mathrm{min}$, a variation MCA between $60^{\circ}$ and $-90^{\circ}$, a mean $P$ wave duration of $34 \pm 6 \mathrm{~ms}$ and amplitude of $0.132 \pm 0.051 \mathrm{mV}$, a mean PR interval duration of $56 \pm 11 \mathrm{~ms}$, a mean QRS complex duration of $35 \pm 7 \mathrm{~ms}$ and amplitude of $0.273 \pm 0.269 \mathrm{mV}$, a mean QT interval duration $130 \pm 26 \mathrm{~ms}$, ST segment was isoelectric (13 animals) and with elevation (six animals) and a mean T wave amplitude of $0.19 \pm 0.083 \mathrm{mV}$ and positive. There was no significant difference between males and females. Thus, the electrocardiographic parameters obtained in our study in marmosets can be used as a reference values to in other future researches, providing researchers electrocardiographic parameters that contribute with the literature.
\end{abstract}

INDEX TERMS: Primate, marmoset, Callithrix penicillata, cardiology, electrocardiography.

RESUMO.- 0 conhecimento de parâmetros cardíacos em animais modelo experimentais é essencial para a investigação médica comparativa. Nosso estudo teve como objetivo estabelecer parâmetros eletrocardiográficos e valores de referência para saguis-de-tufo-preto (Callithrix penicillata). Dezenove saguis-de-tufo-preto adultos saudáveis foram utilizados. Uma combinação de tiletamina e zolazepam foi empregada para contenção química antes da realização do exame eletrocardiográfico (ECG). Foi utilizado para o exame de ECG um equipamento computadorizado. Os valores dos parâmetros do ECG encontrados foram frequência cardíaca média de $264 \pm 74$ bpm, uma variação do eixo cardí-

\footnotetext{
${ }^{1}$ Recebido em 8 de dezembro de 2012.

Aceito para publicação em 2 de maio de 2013.

2 Departamento de Medicina Veterinária, Universidade Federal do Paraná (UFPR), Rua dos Funcionários 1540, Curitiba, PR 80035-050, Brasil. *Autor para correspondência: amaliagiannico@uol.com.br

${ }^{3}$ Departamento de Medicina Veterinária, Universidade do Estado de Santa Catarina (UDESC), Av. Luiz de Camões 2090, Lages, SC 88520-000, Brasil.
}

aco médio entre $60^{\circ}$ e $-90^{\circ}$, a duração da onda P média de $34 \pm 6 \mathrm{~ms}$ e amplitude de $0,132 \pm 0,051 \mathrm{mV}$, um intervalo PR com duração de $56 \pm 11 \mathrm{~ms}$, duração média do complexo QRS de $35 \pm 7 \mathrm{~ms}$ e amplitude de $0,273 \pm 0,269 \mathrm{mV}$, duração do intervalo QT de $130 \pm 26 \mathrm{~ms}$, segmento ST isoelétrico (13 animais) e com supradesnível (seis animais) e uma amplitude de onda T de $0,19 \pm 0,083 \mathrm{mV}$ e com polaridade positiva. Não houve diferença significativa entre machos e fêmeas. Os parâmetros eletrocardiográficos obtidos em nosso estudo em saguis-de-tufo-preto podem ser utilizados como referência em outras pesquisas futuras, oferecendo aos pesquisadores parâmetros eletrocardiográficos que contribuem com a literatura.

TERMOS DE INDEXAÇÃO: Primata, saguis-de-tufo-preto, Callithrix penicillata, cardiologia, eletrocardiograma.

\section{INTRODUÇÃO}

Os primatas em geral apresentam a anatomia cardíaca e vascular muito semelhante às dos seres humanos e tem 
sido utilizados há longa data como modelos animais experimentais para estudos cardiovasculares (Goldberg \& Moberg 1985, Hassimoto et al. 2002, Ludlage \& Mansfield 2003).

Os saguis (gênero Callithrix) são primatas do novo mundo originários do Brasil, filogeneticamente muito próximos aos seres humanos (Horii et al. 2002) e tem sido utilizados para estudos biomédicos desde a década de 1960 (Ludlage \& Mansfield 2003, Cyranoski 2009). Historicamente, os primeiros modelos experimentais para avaliação da fisiologia cardíaca foram os primatas do velho mundo, como exemplo o macaco rhesus (Macaca mulatta) (Goldberg \& Moberg 1985) que ainda nos dias atuais é considerado um bom modelo experimental para avaliação eletrofisiológica (Hassimoto et al. 2002). Todavia, sua baixa prolificidade, associada ao grande porte dos animais e às dificuldades de manejo, tornaram seu uso dispendioso. 0 uso de saguis e primatas do novo mundo em estudos cardiológicos tem aumentado ao longo dos anos (Brady et al. 2003, Senos 2010), por se tratar de uma alternativa mais viável que a utilização de outros primatas não-humanos. A escolha de saguis (Callithrix sp.) apresenta certas vantagens devido ao pequeno porte dos animais, relativo baixo custo, fácil manejo e por produzirem até quatro crias anualmente (Benirschke \& Richart 1960, Hampton Junior et al. 1966, Epple 1970, Cyranoski 2009, Orsi et al. 2011, Shimazawaa et al. 2013, Tardif et al. 2013).

O conhecimento de parâmetros cardiovasculares nos animais empregados como modelos experimentais é essencial tanto para a pesquisa médica quanto veterinária, além de ser de grande valia para cuidados médicos veterinários específicos (Ludlage \& Mansfield 2003), principalmente quando se considera a utilização destes animais para este fim (Mansfield 2003).

Para que se consiga realizar um diagnóstico cardiológico mais preciso, é essencial a compreensão das características cardíacas normais, entre elas a eletrofisiológica, dos animais selvagens assim como dos de estimação não convencionais. A literatura cardiológica em saguis traz trabalhos relacionados à pressão arterial e frequência cardíaca (Gerber, Schnell \& Anzenberger 2000), doenças cardiovasculares que incluem a ocorrência de fibrose do miocárdio (Chalmers et al. 1983), miocardite crônica (Tucker 1994), dilatação ventricular (Okazaki et al. 1996), e fibrilação ventricular (Brady et al. 2003). Não existem informações precisas quanto à avaliação eletrocardiológica em saguis (Callithrix sp.) e dados sobre os parâmetros eletrocardiográficos em primatas do novo mundo são escassos na literatura médica.

Como forma de contribuir com a literatura sobre cardiologia comparada, que vem recebendo crescente interesse nas duas últimas décadas, o presente estudo objetivou estabelecer parâmetros eletrocardiográficos normais, gerando valores de referência para saguis-de-tufo-preto (Callithrix penicillata). Além disso, esses dados podem fornecer ferramentas que auxiliem em inferências a respeito de eventuais condições patológicas nos animais dessa espécie, contribuindo ainda para o estabelecimento de primatas neotropicais como modelos experimentais das pesquisas biomédicas.

\section{MATERIAL E MÉTODOS}

Animais. Este estudo foi aprovado pelo Instituto Chico Mendes de Conservação da Biodiversidade - ICMBIO por meio da autorização para atividades com finalidade científica número 23065-2 e pela Comissão de Ética no Uso de Animais de Setor de Ciências Agrárias da Universidade Federal do Paraná, protocolo 015/2010. Foi utilizado nesta pesquisa um total de 19 saguis-de-tufo-preto (Callithrix penicillata), considerados adultos jovens, com idade média estimada de três anos, sendo 11 fêmeas e oito machos, pesando entre 180 e 420 gramas (média de 305 gramas) atendidos pelo serviço de clinica médica e cirúrgica de animais selvagens do hospital veterinário.

Procedimentos de contenção/imobilização. Previamente ao exame eletrocardiográfico os animais foram submetidos à contenção física realizada por um profissional com o uso de luvas de couro, recebendo consecutivamente anestesia injetável por via intramuscular de uma associação de tiletamina e zolazepam (Zoletil, Laboratório Virbac do Brasil, São Paulo, SP, Brasil) na dose de 7,5mg/kg como método de contenção farmacológica para a realização do exame eletrocardiográfico (ECG). Após a aplicação da associação anestésica, o tempo médio de indução foi de $10 \mathrm{mi}$ nutos com duração média da anestesia de 30 minutos. Após este período, os animais iniciavam sinais de recuperação anestésica, que perdurou aproximadamente duas horas consecutiva, até a completa recuperação.

Exame eletrocardiográfico. O ECG foi realizado com o aparelho de eletrocardiograma computadorizado ECGPC (TEB - Tecnologia Eletrônica Brasileira Ltda., São Paulo, SP, Brasil), os eletrodos utilizados foram do tipo jacaré sem dentes, onde os dentes foram desgastados com lima, e de menor tamanho para melhor conforto dos animais. Estes foram fixados diretamente na pele do animal, fazendo-se necessário o uso de álcool para permitir melhor condução do estímulo elétrico. Durante a realização do exame os animais permaneceram em decúbito dorsal com os eletrodos craniais colocados na altura da articulação escápulo-umeral, e os caudais dispostos na região da prega cutânea inguinal, próximo ao membro pélvico, seguindo-se o seguinte padrão: eletrodo amarelo no membro torácico esquerdo, eletrodo vermelho no mem-

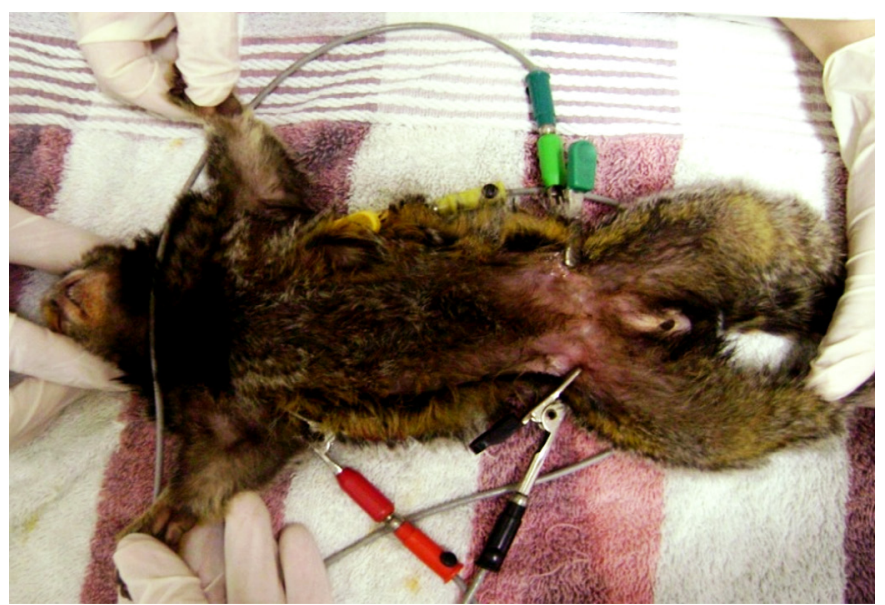

Fig.1. Disposição dos eletrodos em um sagui-de-tufo-preto (Callithrix penicillata) para a realização do exame eletrocardiográfico. Eletrodos craniais colocados na altura da articulação escápula-umeral, e caudais permaneceram dispostos na prega inguinal próximo ao membro pélvico, seguindo-se a seguinte ordem: eletrodo amarelo no membro torácico esquerdo, eletrodo vermelho no membro torácico direito, eletrodo verde no membro pélvico esquerdo e o eletrodo preto no membro pélvico direito. 
bro torácico direito, eletrodo verde no membro pélvico esquerdo e o eletrodo preto no membro pélvico direito (Fig.1).

Os traçados eletrocardiográficos foram gravados durante dois a três minutos, registrando-se seis derivações eletrocardiográficas em cada animal (derivações I, II, III, aVR, aVL e aVF), sendo a derivação II escolhida para análise. Na interpretação dos traçados foram analisados a frequência cardíaca (FC), eixo cardíaco médio (ECM), duração da onda P, complexo QRS, intervalos PR e QT (em milissegundos), e amplitude das ondas $\mathrm{P}, \mathrm{R}$ e $\mathrm{T}$ (em $\mathrm{mV}$ ), bem como do segmento ST.

Exame radiográfico. Foram realizadas radiografias do tórax nas projeções ventrodorsal e lateral direita utilizando o aparelho móvel da marca CRX, modelo $100 \mathrm{~mA} / 100 \mathrm{Kv}$ TRAF. As radiografias foram analisadas sistematicamente quanto à presença de alterações na silhueta cardíaca, campos pulmonares e estruturas extrapulmonares que eventualmente pudessem causar alterações ou desvios do eixo cardíaco.

Análises estatísticas. Foi realizada análise estatística descritiva e inferencial utilizando o programa StatView (SAS, Cary, NC). Para comparação dos dados eletrocardiográficos entre os sexos foi empregado o teste- $t$ não-pareado. Valores de $P<0,05$ foram considerados significativos.

\section{RESULTADOS}

A FC variou de 112 a 439 batimentos por minuto (bpm) (média de $264 \pm 74$ bpm), apresentando ritmo sinusal em todos os traçados (Fig.2). O ECM apresentou variações en-

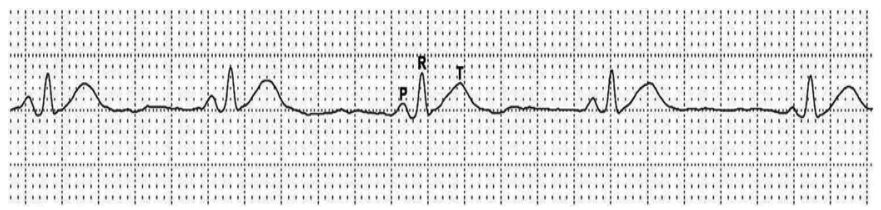

Fig.2. Traçado eletrocardiográfico na derivação DII, N e $50 \mathrm{~mm} / \mathrm{s}$ de um sagui-de-tufo-preto (Callithrix penicillata). Interpretação do exame eletrocardiográfico: ritmo sinusal normal; frequencia cardíaca, 120 batimentos por minuto; onda P, 0,12mV x 45ms; intervalo PR, $50 \mathrm{~ms}$; complexo QRS, $0,34 \mathrm{mV}$ x $40 \mathrm{~ms}$; intervalo QT, $170 \mathrm{~ms}$; segmento ST, isoelétrico; onda T, positiva, $0,25 \mathrm{mV}$.

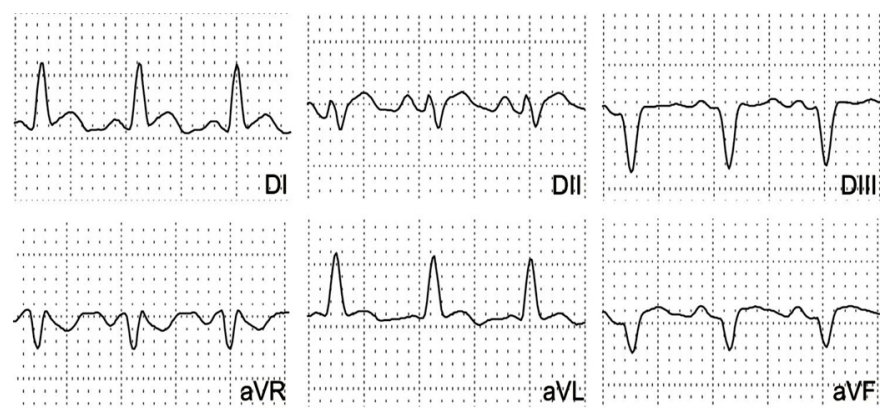

Fig.3. Traçado eletrocardiográfico de um sagui-de-tufo-preto (Callithrix penicillata) em $50 \mathrm{~mm} / \mathrm{s}$ e N. Eixo cardíaco médio encontra-se entre $-30^{\circ}$ e $0^{\circ}$. tre os indivíduos, porém permaneceu sempre deslocado para a esquerda. Do total de 19 animais, sete apresentaram ECM entre -30 e $0^{\circ}$ (Fig. 3 ), seis entre $+30^{\circ}$ e $+60^{\circ}$, quatro entre $-60^{\circ} \mathrm{e}-30^{\circ}$, um animal entre $0^{\circ} \mathrm{e}+30^{\circ}$ e outro entre $-90^{\circ}$ e $-60^{\circ}$.

O Quadro 1 resume os parâmetros eletrocardiográficos avaliados. Não houve qualquer diferença significativa nestes parâmetros entre machos e fêmeas (Quadro 2).

\section{Quadro 1. Média e desvio padrão dos parâmetros eletrocardiográficos de saguis-de-tufo-preto (Callithrix penicillata)}

\begin{tabular}{lcc}
\hline \multicolumn{1}{c}{ Deflexões ECG } & Média \pm SD & Variação \\
\hline Onda P - duração (ms) & $34 \pm 6$ & $23-43$ \\
Segmento PR - duração (ms) & $56 \pm 11$ & $40-73$ \\
Complexo QRS - duração (ms) & $35 \pm 7$ & $23-50$ \\
Intervalo QT - duração (ms) & $130 \pm 26$ & $73-177$ \\
Onda P - amplitude (mV) & $0,132 \pm 0,051$ & $0,05-0,3$ \\
Onda R - amplitude (mV) & $0,273 \pm 0,269$ & $0,02-0,82$ \\
Onda T - amplitude (mV) & $0,19 \pm 0,083$ & $0,09-0,45$
\end{tabular}

Onda P. As ondas $\mathrm{P}$ observadas são pequenas, monofásicas e arredondadas, com polaridade positiva nas derivações I, II, III, aVL e aVF, e negativa na derivação aVR. A amplitude média foi de $0,132 \pm 0,051 \mathrm{mV}$ e o intervalo desta onda teve duração média de $34 \pm 6 \mathrm{~ms}$.

Intervalo PR e QT. Os intervalos PR tiveram média de $56 \pm 11 \mathrm{~ms}$. Na fase de repolarização ventricular, observou-se um intervalo QT de $130 \pm 26$ ms que diminui com o aumento da frequência cardíaca.

Segmento ST. O segmento ST da maioria dos saguis-de-tufo-preto (13 animais) foi isoelétrico, e em seis animais observou-se um supradesnível que teve a maior amplitude de $0,15 \mathrm{mV}$.

Complexo QRS. Os complexos QRS tiveram amplitude média de $0,273 \pm 0,269 \mathrm{mV}$ e a duração média de $35 \pm 7 \mathrm{~ms}$, com onda R positiva nas derivações I, II, III, aVL e aVF, e negativa na derivação aVR.

Onda T. A morfologia da onda T foi arredondada e ligeiramente assimétrica, observando-se apenas ondas positivas, com amplitude média de $0,19 \pm 0,083 \mathrm{mV}$.

A avaliação radiográfica do tórax possibilitou a exclusão de eventuais massas e linfonodomegalia, e descartou alterações em campos pulmonares que deslocassem a silhueta cardíaca do seu eixo, já que tais fatos poderiam influenciar a análise do eletrocardiograma.

\section{DISCUSSÃO}

Vários estudos eletrocardiográfico foram realizados em diversas espécies de primatas não-humanos (Malinow 1966, Malinow \& Delannoy 1967, Malhotra et al. 1975, Bellinger et al. 1980, Atkins \& Dickie 1986, Liang et al. 2005), não

Quadro 2. Parâmetros eletrocardiográficos em machos e fêmeas de saguis-de-tufo-preto (Callithrix penicillata)

\begin{tabular}{|c|c|c|c|c|c|c|c|c|c|}
\hline Sexo & $\begin{array}{l}\text { Número de } \\
\text { animais }\end{array}$ & $\begin{array}{c}\text { Frequencia } \\
\text { cardíaca } \\
\text { (bpm) }\end{array}$ & $\begin{array}{c}\text { Duração } \\
\text { onda P } \\
\text { (ms) }\end{array}$ & $\begin{array}{c}\text { Intervalo } \\
\text { PR (ms) }\end{array}$ & $\begin{array}{c}\text { Duração } \\
\text { complexo } \\
\text { QRS (ms) }\end{array}$ & $\begin{array}{c}\text { Intervalo } \\
\text { QT (ms) }\end{array}$ & $\begin{array}{c}\text { Amplitude } \\
\text { onda P (mV) }\end{array}$ & $\begin{array}{c}\text { Amplitude } \\
\text { complexo } \\
\text { QRS (mV) }\end{array}$ & $\begin{array}{c}\text { Amplitude } \\
\text { onda T (mV) }\end{array}$ \\
\hline Fêmea & 11 & $264 \pm 73^{a}$ & $35 \pm 7$ & $57 \pm 11$ & $33 \pm 6$ & $124 \pm 29$ & $0,126 \pm 0,037$ & $0,295 \pm 0,269$ & $0,180 \pm 0,096$ \\
\hline Macho & 8 & $263 \pm 81$ & $33 \pm 6$ & $56 \pm 11$ & $37 \pm 9$ & $137 \pm 22$ & $0,140 \pm 0,069$ & $0,244 \pm 0,283$ & $0,204 \pm 0,063$ \\
\hline
\end{tabular}

Média \pm SD. 
havendo, todavia, estudos eletrocardiográficos em saguis-de-tufo-preto. Whelan et al. (1999) descreveram o monitoramento eletrocardiográfico destes animais sob anestesia sendo utilizado apenas dois eletrodos, com o intuito de observar apenas a presença de arritmias e a frequência cardíaca durante o procedimento anestésico.

A literatura contribui com diferentes dados sobre a FC média dos saguis. Schnell e Wood (1995) obtiveram como valores normais para a FC em Callithrix jacchus que não foram submetidos ao estresse de $153 \pm 4 \mathrm{bpm}$ durante o dia e $220 \pm 6 \mathrm{bpm}$ durante a noite. Michel et al. (1984) observaram que a FC era menor em saguis-de-tufo-preto livres do que em saguis-de-tufo-preto sob contenção, que foi de $348 \pm 51 \mathrm{bpm}$. Nossa pesquisa obteve FC média de $264 \pm 74$ bpm, variando entre 112 e $439 \mathrm{bpm}$. Existe uma série de fatores envolvidos na variação da FC nestes animais, entre eles o estresse de contenção, peso médio do grupo, idade e estado metabólico (anestesiados ou não). Uma comparação interespecífica pode ilustrar a diferença de peso observada entre primatas de diferentes tamanhos como macacos japoneses (Macaca cyclopis) (126 bpm) (Liang et al. 2005) e macacos rhesus (257 bpm) em ambas espécies sem anestesia (Malinow 1966) e macacos cynomolgus (Macaca fascicularis) anestesiados com cetamina (182 bpm) (Atkins \& Dickie 1986). Isso pode estar relacionado ao tamanho relativamente menor dos saguis-de-tufo-preto, que apresentaram peso médio de $305 \mathrm{~g}$, em comparação com outros relatos de macacos rhesus $(4,9 \mathrm{~kg})$ (Malinow 1966), macacos cynomolgus $(3,2 \mathrm{~kg}$ ) (Atkins \& Dickie 1986), e macacos japoneses (6,8 kg) (Malinow \& Delannoy 1967). Outra razão pode ter sido o efeito da anestesia (Bellinger et al. 1980), mas mais estudos para avaliar os efeitos de diferentes agentes anestésicos em vários primatas não-humanos são necessários.

A presença de ondas $P$ apresentando alta amplitude não é incomum em primatas não-humanos (Liang et al. 2005) e foi relatada em $40 \%$ de macacos rhesus (Malinow 1966), em 37\% de macacos japonês (Malinow \& Delannoy 1967) e comumente em macacos cynomolgus (Toback et al. 1978), porém, não foi observada em nosso estudo, possivelmente pelos saguis-de-tufo-preto apresentarem menor tamanho e peso.

O intervalo PR descrito em outros primatas varia de 70ms para o macacos rhesus (Malinow 1966), a $80 \mathrm{~ms}$ para os macacos cynomolgus (Atkins \& Dickie 1986), macacos-da-face-vermelha (Macaca arctoides) (Malhotra et al. 1975), e macacos japoneses (Malinow \& Delannoy 1967). Neste estudo, a média encontrada para os saguis-de-tufo-preto para o mesmo intervalo foi de $56 \mathrm{~ms}$, sendo atribuído esta diferença ao menor peso corporal dos indivíduos, associado à frequencia cardíaca mais elevada. 0 mesmo ocorreu para o intervalo QT (média de $130 \mathrm{~ms}$ ), sendo descrito para outros primatas uma variação de $140 \mathrm{~ms}$ em macacos rhesus (Malinow 1966), a 200ms em macacos cynomolgus (Atkins \& Dickie 1986) e macacos japoneses (Malinow \& Delannoy 1967), e $270 \mathrm{~ms}$ em macacos-da-face-vermelha (Malhotra et al. 1975).

Alguns animais apresentaram complexo QRS com morfologia semelhante ao encontrado em pessoas e em outros primatas não-humanos (Malinow 1966, Malinow \& Delannoy 1967, Atkins \& Dickie 1986), contudo observou um predomínio de complexos de baixa amplitude nos animais. O ECM apresentou variação de $-90^{\circ}$ a $60^{\circ}$, diferente dos demais primatas descritos na literatura como macacos rhesus e macacos japonês, que apresentam variação do ECM entre $50^{\circ}$ e $100^{\circ}$ (Liang et al. 2005).

O supradesnível do segmento ST observado em alguns animais é provavelmente uma variantes normal para esta espécie, mas um inespecífico distúrbio de repolarização ventricular não pode ser excluído. A onda T positiva foi observada em todos os animais, semelhante ao encontrado em seres humanos. Inversão da onda T ou outras alterações em sua morfologia não foi observada neste estudo, diferente do encontrado por outros autores em macacos japoneses (Liang et al. 2005).

Os saguis-de-tufo-preto deste estudo, bem como o observado por Liang et al. (2005) em macacos japoneses, não apresentaram traçados eletrocardiográficos anormais, exemplo a presença de arritmias, como complexos ventriculares prematuros, encontrados em outros primatas não-humanos (Malinow 1966, Malhotra et al. 1975, Toback et al. 1978).

A avaliação do traçado eletrocardiográfico nos saguis-de-tufo-preto mostrou-se semelhante ao padrão eletrocardiográfico de seres humanos, evidenciando a importância destes animais na pesquisa como modelo experimental para estudos cardiológicos. Acredita-se que a obtenção dos valores eletrocardiográficos nos saguis-de-tufo-preto (Callithrix penicillata) deste estudo pode ser empregada futuramente como referência. Ainda sim, o uso destes dados poderá fornecer informações aos médicos veterinários clínicos de animais selvagens auxiliando em uma maior compreensão sobre a fisiologia cardíaca na saúde e na doença.

\section{REFERÊNCIAS}

Atkins C.E. \& Dickie B.C. 1986. Electrocardiogram of the clinically normal, ketamine sedated Macaca fascicularis. Am. J. Vet. Res. 47(2):455-457.

Bellinger D., Greene A.W. \& Corbett W.T. 1980. Electrocardiographic studies in African green monkeys (Cercopithecus aethiops). Lab. Anim. Sci. 30(5):854-859.

Benirschke K. \& Richart R. 1960. Spontaneous acute toxoplasmosis in a marmoset monkey. Am. J. Trop. Med. Hyg. 9(3):269-273.

Brady A.G., Watford J.W., Massey C.V., Rodning K.J., Gibson G.V., Williams l.E. \& Abee C.R. 2003. Studies of heart disease and failure in aged female squirrel monkeys (Saimiri sp.). Comp. Med. 53(6):657-662.

Chalmers D.T., Murgatroyd L.B. \& Wadsworth P.F. 1983. A survey of the pathology of marmosets (Callithrix jacchus) derived from a marmoset breeding unit. Lab. Anim. 17(4):270-279.

Cyranoski D. 2009. Marmoset model takes centre stage: Newly created transgenic primate may become an alternative disease model to rhesus macaques. Nature 459(7246):492.

Epple G. 1970. Maintenance breeding and development of marmoset monkeys in captivity. Folia Primatol. 12(1):56-76.

Gerber P., Schnell C.R. \& Anzenberger G. 2000. Cardiovascular parameters telemetrically measured during pregnancy, parturition, and lactation in a common marmoset (Callithrix jacchus). Contemp. Top. Lab. Anim. Sci. 39(5):14-17.

Goldberg J.M. \& Moberg G.P. 1985. Autonomic control of heart rate in the neonatal rhesus monkey. J. Med. Primatol. 14(1):19-27.

Hampton Junior J.K., Hampton S.H. \& Landwehr B.T. 1966. Observations 
on a successful breeding colony of the marmoset. Oedipomidas oedipus. Folia Primatol. 4(4):265-287.

Hassimoto M., Harada T., Kaga N., Murano H. \& Obata M. 2002. Accurate evaluation of QT interval in conscious rhesus monkeys (Macaca mulatta) by use of Holter ECG. J. Electrocardiol. 35(4):333-342.

Horii I., Kito G., Hamada T., Jikuzono T., Kobayashi K. \& Hashimoto K. 2002. Development of telemetry system in the common marmoset-cardiovascular effects of astemizole and nicardipine. J. Toxicol. Sci. 27(2): 123-130.

Liang S., Chin S. \& Yeh L. 2005. Electrocardiographic studies in Formosan Macaques (Macaca cyclopis). Zool. Stud. 44(4):462-467.

Ludlage E. \& Mansfield K. 2003. Clinical care and diseases of common marmoset (Callithrix jacchus). Comp. Med. 53(4):369-382.

Malhotra V., Pick R., Pick A. \& Glick G. 1975. Electrocardiographic studies in the stumptail macaque (Macaca arctoides). J. Electrocardiol. 8(3): 247-251.

Malinow M.R. \& Delannoy C.W. 1967. The electrocardiogram of Macaca fuscata. Folia Primatol. 7(3):284-291.

Malinow M.R. 1966. An electrocardiographic study of Macaca mulatta. Folia Primatol. 4(1):51-65.

Mansfield K. 2003. Marmosets models commonly used in biomedical research. Comp. Med. 53(4):383-392.

Michel J.B., Wood J., Hofbauer K., Corvol P. \& Menard J. 1984. Blood pressure effects of renin inhibition by human renin antiserum in normotensive marmosets. Am. J. Physiol. 246(3):309-316.

Okazaki Y., Kurata Y., Makinodan F., Kidachi F., Yokoyama M., Wako Y., Ya- magishi Y., Katsuta O., Takechi M. \& Tsuchitani M. 1996. Spontaneous lesions detected in the common cotton-eared marmosets (Callithrix jacchus). J. Vet. Med. Sci. 58(3):181-190.

Orsi A., Rees D., Andreini I., Venturella S., Cinelli S. \& Oberto G. 2011. Overview of the marmoset as a model in nonclinical development of pharmaceutical products. Regul. Toxicol. Pharmacol. 59(1):19-27.

Schnell C.R. \& Wood J.M. 1995. Measurement of blood pressure and heart rate by telemetry in conscious unrestrained marmosets. Lab. Anim. 29(3):258-261.

Senos R. 2010. Estudo morfofuncional de corações de Callithrix jacchus. Dissertação de Mestrado em Anatomia dos Animais Domésticos e Silvestres, Faculdade de Medicina Veterinária e Zootecnia, Universidade de São Paulo, São Paulo, SP. 66p.

Shimazawa M., Nakamura S., Miwa M., Tsuruma K., Aihara M., Nakamura K. \& Hara H. 2013. Establishment of the ocular hypertension model using the common marmoset. Exp. Eye. Res. 22(111):1-8.

Tardif S.D., Power M.L., Ross C.N. \& Rutherford J.N. 2013. Body mass growth in common marmosets: toward a model of pediatric obesity. Am. J. Phys. Anthropol. 150(1):21-8.

Toback J.M., Clark J.C. \& Moorman W.J. 1978. The electrocardiogram of Macaca fasicularis. Lab. Anim. Sci. 28(2):182-185.

Tucker M.J. 1984. A survey of the pathology of marmosets (Callitrix jacchus) under experiment. Lab. Anim. 18(4):351-358.

Whelan G., James M.F., Samson N.A. \& Wood N.I. 1999. Anaesthesia of the common marmoset (Callithrix jacchus) using continuous intravenous infusion of alphaxalone/alphadalone. Lab. Anim. 33(1):24-29. 